\title{
Performance Improvement of Joint Source-Channel Coding with Unequal Power Allocation
}

\author{
Jiguang He, Yong Li, Guangfu Wu, Shen Qian, Qiang Xue, and Tad Matsumoto
}

\begin{abstract}
We develop a joint source-channel coding (JSCC) scheme based on an unequal power allocation strategy, in which different levels of power are allocated according to a priori probability distribution of the information bits. Two protograph low-density parity-check codes are used as the source code and the channel code, respectively, in the proposed JSCC scheme. With fixed row weight and row element alphabet, optimization on the source code is conducted via random search to obtain a bestfound base matrix with the lowest decoding threshold among all the searched base matrices. Simulation results show that the new JSCC scheme outperforms the traditional equal power allocation based JSCC by exploiting the source statistics at the decoder. Moreover, theoretical threshold analysis is in agreement with the practical simulation results.
\end{abstract}

Index Terms-Joint protograph extrinsic information transfer (JPEXIT), joint source-channel coding (JSCC), protograph low-density parity-check (LDPC) codes, unequal power allocation (UPA)

\section{INTRODUCTION}

Joint source-channel coding (JSCC) has been intensively investigated in [1]-[5], which achieved significant gains compared with its conventional counterpart, i.e., separate sourcechannel coding (SSCC). To be specific, the advantages of JSCC over SSCC lie in: (1) The residual redundancy left by the source encoder can be further exploited by the channel encoder/decoder, and even by the modulator/demodulator. (2) JSCC, as a whole, can be viewed as a serially concatenated channel code with soft information exchange between the constituent decoders.

In general, unequal power allocation (UPA) is realized by means of modulation. The significance of the individual bits is taken into account, and more power is potentially allocated to the most significant bit (MSB) while keeping the average transmit power per bit constant [6]. Unlike the method given in [6], another strategy is to conduct the power allocation by taking the probability distribution of the bits

This work is partially supported by China NSF under Grants 61401050 and 11461031

J. He, and Q. Xue are with the Centre for Wireless Communications, University of Oulu, Oulu, 90014, Finland, (e-mail: jiguang.he@oulu.fi, qiang.xue@oulu.fi).

$\mathrm{Y}$. Li is with the Key Laboratory of Mobile Communication, Chongqing University of Posts and Telecommunications, Chongqing, 400065, China, (email: yongli@cqupt.edu.cn).

G. Wu is with the Department of Information Engineering, Jiangxi University of Science and Technology, Jiangxi, 341000, China, (e-mail: wuguangfu@126.com).

S. Qian and T. Matsumoto are with the school of Information Science, Japan Advanced Institute of Science and Technology (JAIST), Ishikawa, 9231292, Japan, and the Centre for Wireless Communications, University of Oulu, Oulu, 90014, Finland, (e-mail: shen.qian@jaist.ac.jp, matumoto@jaist.ac.jp). into consideration [7], [8]. Specifically, the bits with less probability are transmitted with higher power and vice versa.

In this letter, we introduce the methodology of UPA into the protograph low-density parity-check (LDPC) codes assisted JSCC (P-JSCC) system [4], [5] for the transmission of nonuniform binary memoryless sources, where the source and channel codes are both unpunctured protograph LDPC codes. In general, the probability distribution of the output bits from the source encoder is highly likely to be non-uniform and the structure of the protograph can be well exploited in the calculation of the probability distribution. Thus, the UPA could be directly applied to the P-JSCC system with minor modifications at the transmitter and receiver sides. As a consequence, significant performance improvement is achieved compared with the P-JSCC system with equal power allocation (EPA). Furthermore, asymptotic performance is analyzed via joint protograph extrinsic information transfer (JPEXIT), developed in [5], to verify the superiority of the P-JSCC system with UPA over its EPA based counterpart. An attempt for optimizing the source code is introduced via random search while fixing the channel code, which provides a best-found base matrix with the lowest decoding threshold among all the searched base matrices according to the JPEXIT analysis.

\section{SySTEM DESCRIPTION}

The system model of the P-JSCC system with UPA is shown in Fig. 1. Let $\mathbf{S}=\left\{s_{1} s_{2} s_{3} \cdots\right\}$ be a sequence generated by an independent and identically distributed (i.i.d.) non-uniform binary memoryless source with a probability distribution $\left(p_{0}, p_{1}=1-p_{0}\right)$, where $p_{1}=\operatorname{Pr}\left\{s_{i}=1\right\} \neq$ $0.5, i=1,2, \ldots$. The whole transmission chain is described as follows. The non-uniform source sequence $\mathbf{S}$ is compressed by an unpunctured protograph LDPC code and a compressed sequence $\mathbf{U}=\left\{u_{1} u_{2} u_{3} \cdots\right\}$ is obtained, which is sent to the systematic channel encoder as information bits. Then, another unpunctured protograph LDPC code is employed to further encode $\mathbf{U}$ for a reliable transmission. UPA is utilized as the modulation mode for the output sequence of channel encoder $\mathbf{X}=\left\{x_{1} x_{2} x_{3} \cdots\right\}$ due to the non-uniformity of the information bits $\mathbf{U}$. As a consequence, more (less) power is allocated to the information bits that are less (more) likely to occur. However, the power is always equally allocated to the parity bits since their probability distribution is very difficult, if not impossible, to compute. Specifically, the UPA for the information bits is achieved by using a series of 2-ary PAM constellations. These PAM constellations have constant average power and the distance between each pair of the 


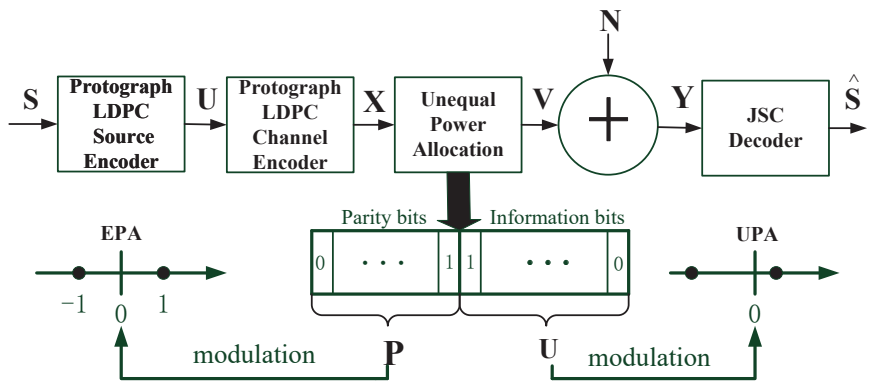

Fig. 1. The system model of P-JSCC system with UPA.

constellation points is greater than 2 . The average power of the information bits and parity bits are both normalized to unity during the transmission. At the receiver side, the received signal has the form $\mathbf{Y}=\mathbf{V}+\mathbf{N}$, where $\mathbf{V}=\left\{v_{1} v_{2} v_{3} \cdots\right\}$ is the modulated symbol sequence and $\mathbf{N}=\left\{n_{1} n_{2} n_{3} \cdots\right\}$ is additive white Gaussian noise (AWGN) with zero mean and $\sigma_{n}^{2}$ variance. The joint source-channel (JSC) decoder runs in parallel over a bipartite Tanner graph describing the JSCC structure, in which the belief propagation (BP) algorithm with a priori knowledge of the source distribution is applied.

\section{P-JSCC System WITH UPA}

In this section, we mainly discuss the different aspects between the P-JSCC system with UPA and the conventional PJSCC system with EPA. The differences lie in the modulation method at the transmitter and the computation of initial channel log-likelihood ratios (LLRs) at the receiver. In reality, the remaining procedures of source and channel coding/decoding still remain unchanged.

\section{A. Source Code}

A protograph LDPC code can be represented by a base matrix, from which larger matrices with various sizes can be obtained by means of a "copy-and-permute" operation [9]. We assume, in this letter, that the base matrix of the source code is of the form

$$
\mathbf{B}_{\mathrm{sc}}=\left[\begin{array}{llll}
b_{1,1} & b_{1,2} & \cdots & b_{1, n_{s c}} \\
b_{2,1} & b_{2,2} & \cdots & b_{2, n_{s c}} \\
\vdots & \vdots & \vdots & \vdots \\
b_{m_{s c}, 1} & b_{m_{s c}, 2} & \cdots & b_{m_{s c}, n_{s c}}
\end{array}\right],
$$

where $m_{s c}$ and $n_{s c}$ are the number of rows and columns, respectively. The corresponding rate is given by $R_{s c}=m_{s c} / n_{s c}$. The entry $b_{i, j}$ in the matrix represents the number of edges that connect the $j$ th variable node and the $i$ th check node. The row weights of $\mathbf{B}_{\mathrm{sc}}$ can be written as

$$
W_{i}=\sum_{j=1}^{n_{s c}} b_{i, j}, \text { for } i=1, \cdots, m_{s c} .
$$

The aforementioned base matrix can be expanded to a larger one by the use of the progressive edge growth (PEG) algorithm [10] and in this way the parity check matrix for the source code (i.e., $\mathbf{H}_{\mathrm{sc}}$ ) with larger size $l \times n$ is obtained, where $l \gg m_{s c}$ and $n \gg n_{s c}$.
The repetition time (i.e., $N$ ) satisfies the equation $N=$ $n / n_{s c}=l / m_{s c}$. By using the "copy-and-permute" operation, the $\mathbf{H}_{\text {sc }}$ has a good structure. That is, every $N$ rows have the same row weight, e.g., the first $N$ rows of $\mathbf{H}_{\text {sc }}$ have the row weight $W_{1}$, the second $N$ rows of the $\mathbf{H}_{\text {sc }}$ have the row weight $W_{2}$, and the rest could be deduced by analogy. The row weights of $\mathbf{H}_{\mathrm{sc}}$ can be summarized by

$$
\begin{gathered}
W((i-1) N+1)=W((i-1) N+2)=\cdots=W(i N)=W_{i}, \\
\text { for } i=1, \cdots, m_{s c},
\end{gathered}
$$

where $W(q)$ denotes the weight of the $q$ th row of $\mathbf{H}_{\text {sc }}$.

\section{B. UPA Modulation Strategy}

The source compression process can be written as $\mathbf{U}=$ $\mathbf{H}_{\mathrm{sc}} \times \mathbf{S}$. Namely, the $i$ th information bit $u_{i}$ is the modulo-2 sum of $W(i)$ source bits associated with $W(i) 1$ 's in the $i$ th row of $\mathbf{H}_{\mathrm{sc}}$. Therefore, $u_{i}$ will be 0 when the $W(i)$ source bits contain an even number of 1's, and 1, otherwise. We introduce a simple algorithm to estimate the probability distribution of $u_{i}$ as follows:

For $i=1, \cdots, l$

1) Define $q_{i}^{0}=\operatorname{Pr}\left(u_{i}=0\right)$ and $q_{i}^{1}=\operatorname{Pr}\left(u_{i}=1\right)$.

2) Initialize $q_{i}^{0}=0$ and $j=0$.

3) Update $q_{i}^{0}=q_{i}^{0}+\left({ }^{W(i)}\right) p_{1}^{j} p_{0}^{(W(i)-j)}$, where the binomial coefficient indicates the number of length- $W(i)$ sequences with $j 1$ 's and $(W(i)-j) 0$ 's.

4) Set $j=j+2$ to guarantee that the number of 1's is still even. If $j \leq W(i)$, then go to Step 3).

5) Compute $q_{i}^{1}=1-q_{i}^{0}$.

End

Following the pseudo program, it is relatively straightforward to figure out that $\mathbf{U}$ can be divided into $m_{s c}$ individual blocks and each block has the same probability distribution because of the good structure of $\mathbf{H}_{\mathrm{sc}}$. Owing to the fact that $W(i)$ is usually small (generally, $W(i) \leq 25$ ) and the source sequence is non-uniform, each block within $\mathbf{U}$ remains non-uniformly distributed. Relying on this observation, we introduce the UPA strategy to the information bits and hence improve the bit error rate (BER) performance of the P-JSCC system.

As for the channel code, its parity check matrix $\mathbf{H}_{\mathrm{cc}}$ is generated from another base matrix $\mathbf{B}_{\mathrm{cc}}$ with size $m_{c c} \times n_{c c}{ }^{1}$. The channel code rate is $R_{c c}=\left(n_{c c}-m_{c c}\right) / n_{c c}$. The channel code that we exploit is systematic, and therefore the codeword has the form $\mathbf{X}=[\mathbf{P} \mid \mathbf{U}]$, where $m$ is the length of $\mathbf{X}$ and $\mathbf{P}$ represents the sequence of parity bits of length $m-l$. The relationship between $m$ and $m-l$ satisfies the equation: $m / n_{c c}=(m-l) / m_{c c}$. We do not apply UPA to the parity bits for the sake of simplicity.

The modulation is summarized as below:

For $i=1, \cdots, m-l$ (i.e., parity bits),

$$
v_{i}=\left\{\begin{aligned}
-1, & \text { if } x_{i}=1, \\
1, & \text { if } x_{i}=0,
\end{aligned} \text { and } E\left[v_{i}^{2}\right]=1 .\right.
$$

\footnotetext{
${ }^{1}$ In this letter, we assume that the same repetition time is applied to the source and channel codes. Therefore, it is not difficult to know $n_{c c}-m_{c c}=$ $m_{s c}$.
} 
For $i=m-l+1, \cdots, m$ (i.e., information bits),

$$
\begin{aligned}
& v_{i}=\left\{\begin{array}{cl}
-\sqrt{q_{i-m+l}^{0} / q_{i-m+l}^{1}}, & \text { if } x_{i}=1, \\
\sqrt{q_{i-m+l}^{1} / q_{i-m+l}^{0}}, & \text { if } x_{i}=0,
\end{array}\right. \\
& E\left[v_{i}^{2}\right]=\frac{q_{i-m+l}^{0}}{q_{i-m+l}^{1}} \times q_{i-m+l}^{1}+\frac{q_{i-m+l}^{1}}{q_{i-m+l}^{0}} \times q_{i-m+l}^{0}=1,
\end{aligned}
$$

where $E\left[v_{i}^{2}\right]$ denotes the average power of $v_{i}$. The distances between the two constellation points corresponding to the information bits are larger than those corresponding to the parity bits. That is, $\left|\sqrt{q_{i-m+l}^{1} / q_{i-m+l}^{0}}-\left(-\sqrt{q_{i-m+l}^{0} / q_{i-m+l}^{1}}\right)\right|=$ $1 / \sqrt{q_{i-m+l}^{1} q_{i-m+l}^{0}}>|1-(-1)|$, if $0 \leq q_{i-m+l}^{1}<0.5$. It means more protection is imposed on the information bits. As presented in [3]-[5], only the information bit nodes are connected to the check nodes of the source decoder, thus more protection on information bits could bring more reliable extrinsic LLR information to the source decoder. Consequently, significant performance improvement is achieved in the waterfall region.

\section{Modification to Joint Decoder}

The initial channel LLRs are closely related to the modulation schemes. Owing to the application of UPA to the information bits only, we separately consider the channel probabilities as follows:

For $i=1, \cdots, m-l$,

$$
p\left(y_{i} \mid x_{i}=1\right) \propto e^{\frac{-\left(y_{i}+1\right)^{2}}{N_{0}}}, \quad p\left(y_{i} \mid x_{i}=0\right) \propto e^{\frac{-\left(y_{i}-1\right)^{2}}{N_{0}}} .
$$

For $i=m-l+1, \cdots, m$,

$$
\begin{aligned}
& p\left(y_{i} \mid x_{i}=1\right) \propto e^{\frac{-\left(y_{i}+\sqrt{q_{i-m+l}^{0} / q_{i-m+l}^{1}}\right)^{2}}{N_{0}}}, \\
& p\left(y_{i} \mid x_{i}=0\right) \propto e^{\frac{-\left(y_{i}-\sqrt{q_{i-m+l}^{1} / q_{i-m+l}^{0}}\right)^{2}}{N_{0}}},
\end{aligned}
$$

where $N_{0}=2 \sigma_{n}^{2}$ and $y_{i}$ is the $i$ th entry of $\mathbf{Y}$. Therefore, the channel LLR (i.e., $L_{c h}(i)$ ) can be calculated as follows:

For $i=1, \cdots, m-l$,

$$
\begin{aligned}
L_{c h}(i) & =\ln \left(p\left(y_{i} \mid x_{i}=0\right) / p\left(y_{i} \mid x_{i}=1\right)\right) \\
& =\ln \left(e^{\frac{-\left(y_{i}-1\right)^{2}}{N_{0}}} / e^{\frac{-\left(y_{i}+1\right)^{2}}{N_{0}}}\right)=\frac{4 y_{i}}{N_{0}} .
\end{aligned}
$$

For $i=m-l+1, \cdots, m$,

$$
\begin{aligned}
L_{c h}(i) & =\ln \left(p\left(y_{i} \mid x_{i}=0\right) / p\left(y_{i} \mid x_{i}=1\right)\right) \\
& =\ln \left(e^{\frac{-\left(y_{i}-\sqrt{q_{i-m+l}^{1} / q_{i-m+l}^{0}}\right.}{N^{2}}} / e^{\frac{-\left(y_{i}+\sqrt{q_{i-m+l}^{0} / q_{i-m+l}^{1}}\right.}{N_{0}}}\right) \\
& =\frac{2 y_{i} \sqrt{q_{i-m+l}^{0} q_{i-m+l}^{1}}+\left(q_{i-m+l}^{0}\right)^{2}-\left(q_{i-m+l}^{1}\right)^{2}}{q_{i-m+l}^{0} q_{i-m+l}^{1} N_{0}} .
\end{aligned}
$$

As shown in Eq. (11), the a priori probability distribution obtained from the source statistics needs to be taken into account when determining the initial LLRs of the information bits. As for the joint decoder, the only difference between the P-JSCC system with UPA and the P-JSCC system with EPA [4], [5] is the computation of the initial LLRs. The same iterative BP algorithm given in [5] can be directly utilized with the source and channel decoders running in a parallel fashion.

\section{Convergence Behavior of P-JSCC with UPA}

The variance of $L_{c h}(i)$, i.e., $\sigma_{c h}^{2}(i)$, is calculated in terms of two subcases:

For $i=1, \cdots, m-l$,

$$
\sigma_{c h}^{2}(i)=8 / N_{0}
$$

For $i=m-l+1, \cdots, m$,

$$
\sigma_{c h}^{2}(i)=2 /\left(q_{i-m+l}^{0} q_{i-m+l}^{1} N_{0}\right) .
$$

The JPEXIT analysis is implemented to estimate the iterative decoding thresholds based on the base matrices of the source and channel codes [5], i.e., $\mathbf{B}_{\mathrm{sc}}$ and $\mathbf{B}_{\mathrm{cc}}$. The difference between JPEXIT procedures of P-JSCC system with UPA and those of P-JSCC system with EPA only lies in the computation of $\sigma_{c h}^{2}(i)$, which determines the mutual information of the AWGN channel. Note that $q_{i}^{0}=q_{j}^{0}$ if the $i$ th row and the $j$ th row have the same row weight according to the algorithm in Section III. It can readily be known from Eqs. (12) and (13) that these equalities are valid: $\sigma_{c h}^{2}(1)=\sigma_{c h}^{2}(2)=\cdots=\sigma_{c h}^{2}(m-l), \sigma_{c h}^{2}(m-l+1)=$ $\sigma_{c h}^{2}(m-l+2)=\cdots=\sigma_{c h}^{2}(m-l+N), \cdots, \sigma_{c h}^{2}(m-N+1)=$ $\sigma_{c h}^{2}(m-N+2)=\cdots=\sigma_{c h}^{2}(m)$. We simply divide $\left\{\sigma_{c h}^{2}(i)\right\}$ into total $n_{c c}-m_{c c}+1$ groups in terms of the different values of the variance, and define $\rho(i), i=1,2, \ldots, n_{c c}-m_{c c}+1$ to be the variance of each group. Herein, the first group corresponds to the parity bits, and the rest are related to the information bits. Accordingly, the initial mutual information of all the variable nodes in $\mathbf{B}_{\mathrm{cc}}$ is expressed as follows:

For $j=1, \ldots, m_{c c}$ (i.e., parity bits),

$$
I_{c c}^{j}=J(\rho(1)) .
$$

For $j=m_{c c}+1, \ldots, n_{c c}$ (i.e., information bits),

$$
I_{c c}^{j}=J\left(\rho\left(j-m_{c c}+1\right)\right)
$$

where $I_{c c}^{j}$ represents the initial mutual information of the $j$ th variable node in $\mathbf{B}_{\mathrm{cc}}$ and a simplified polynomial approximation of function $J(\cdot)$ is available in [11]. The remaining procedures of JPEXIT for the P-JSCC system with UPA are omitted due to the limited space.

\section{OPTIMIZED JSCC}

The row weights of source code not only affect the probability distribution of its output bits (such a probability distribution will affect the decoding threshold accordingly) but also have a great impact on the error floor performance. In principle, higher row weight results in lower error floor but worse waterfall performance, and vice versa [3]. Thus, there must exist a trade-off between the error floor performance and decoding threshold. Our optimization is conducted by fixing the base matrix of the channel code, while obtaining the best-found base matrix of the source code that results in the best waterfall performance by using JPEXIT analysis. Joint optimization of the source and channel codes is a potential topic for further investigation. 
TABLE I

TheORETICAL DECODING THRESHOLDS OF THE P-JSCC SYSTEM WITH UPA AND THE P-JSCC SYSTEM WITH EPA FOR $p_{1}=0.02, R_{s c}=0.25, R_{c c}=0.5$ OVER AWGN CHANNELS

\begin{tabular}{|c|c|c|}
\hline $\mathbf{B}_{\text {sc }}$ & P-JSCC System with UPA & P-JSCC System with EPA \\
\hline Case 1 & $-2.13 \mathrm{~dB}$ & -0.94 \\
\hline Case 2 & $-1.48 \mathrm{~dB}$ & -0.64 \\
\hline Case 3 & $-0.95 \mathrm{~dB}$ & -0.36 \\
\hline
\end{tabular}

The source and channel code rates are set to be 0.25 and 0.5 , respectively. The base matrix of the channel code that we employ is from [9],

$$
\mathbf{B}_{\mathrm{cc}}=\left[\begin{array}{cccccccc}
1 & 0 & 0 & 1 & 4 & 2 & 0 & 0 \\
0 & 1 & 0 & 1 & 4 & 0 & 1 & 1 \\
1 & 0 & 1 & 0 & 4 & 1 & 0 & 0 \\
0 & 1 & 1 & 1 & 4 & 0 & 2 & 2
\end{array}\right]
$$

Different row weights are taken into consideration. Here, we study three cases of $\left.\mathbf{B}_{\mathrm{sc}}{ }^{2}: 1\right) W_{1}=W_{2}=W_{3}=W_{4}=18,2$ ) $\left.W_{1}=W_{2}=W_{3}=W_{4}=14,3\right) W_{1}=W_{2}=W_{3}=W_{4}=$ 10. Furthermore, we restrict the row entries of the base matrix to be chosen from the alphabet $\{0,1,2\}$. By random search ${ }^{3}$, we get the following three best-found solutions associated with the three cases

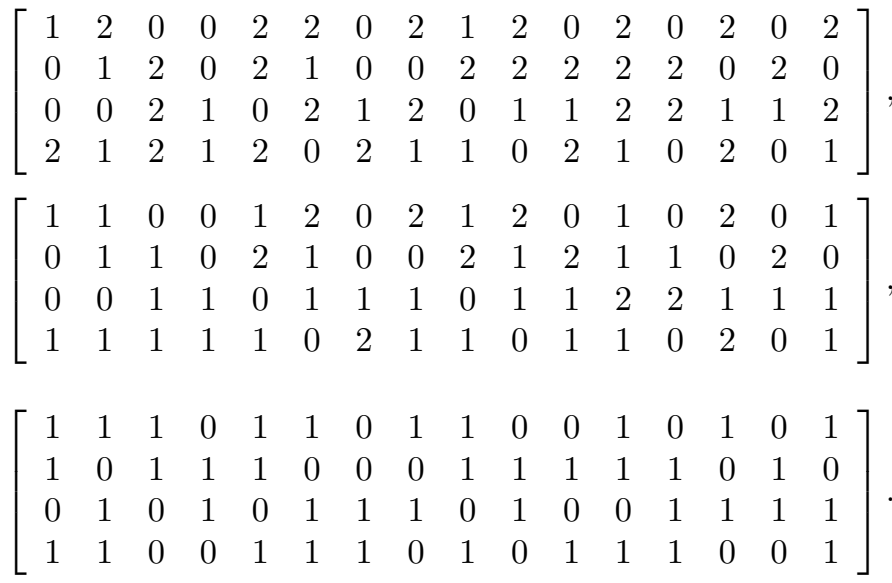

The decoding thresholds (i.e., $\left(E_{b} / N_{0}\right)_{t h}$ with $E_{b}$ denoting the average energy per bit of $\mathbf{S}$ ) of the P-JSCC system with UPA and the P-JSCC system with EPA are shown in Table I, which validates the statement that the lower the row weight, the smaller the decoding threshold.

\section{Simulation Results}

In this section, both practical and theoretical results are provided to demonstrate the benefits of introducing the UPA to the P-JSCC system. The simulation results are given in Fig. 2, where the BER is measured as a function of $E_{b} / N_{0}$ after 50 iterations. The length of the input source sequences is set to be 6400 bits. Comparison is made among the PJSCC system with UPA, the P-JSCC system with EPA, and that given in [3]. We observe that the P-JSCC system with

\footnotetext{
${ }^{2}$ Better performance can be achieved by considering irregular source code and/or increasing the alphabet size of the source base matrix.

${ }^{3}$ Even though we fix the alphabet for each row, exhaustive search consumes huge amount of time.
}

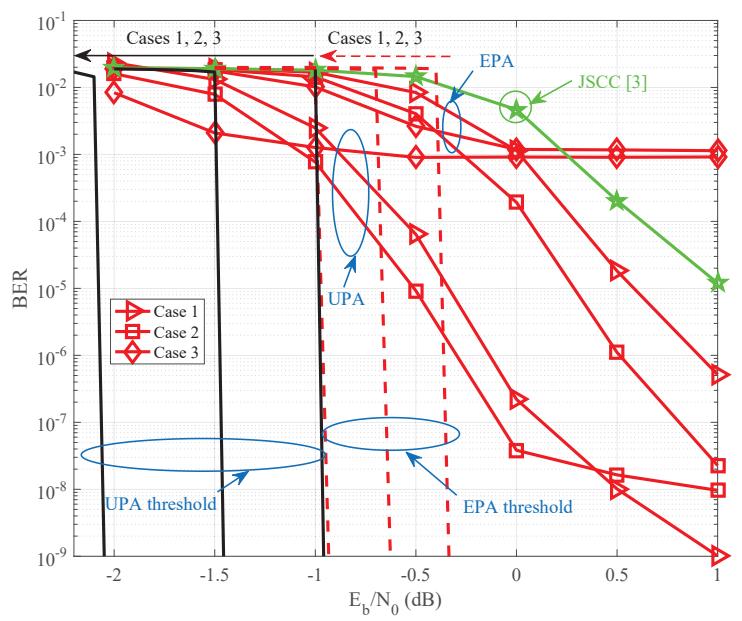

Fig. 2. BER and theoretical decoding threshold as a function of $E_{b} / N_{0}$ for P-JSCC system with UPA and P-JSCC system with EPA, $n=6400$.

UPA performs approximately $0.8 \mathrm{~dB}$ better than that with EPA at BER of $10^{-5}$ for Cases 1 and 2, which is in agreement with the theoretical results. As observed from the simulation results in Fig. 2, error floor appears at BER of $2 \times 10^{-3}$ for the Case 3 due to the small row weight of the source code. However, in theory Case 3 has the smallest decoding threshold, i.e., best waterfall performance. Considering both waterfall performance and error floor performance, the optimal row weight should fall in the region $[10,18]$ with the predefined constraint of the row element alphabet.

\section{REFERENCES}

[1] C. Poulliat, D. Declercq, C. Lamy-Bergot, and I. Fijalkow, "Analysis and optimization of irregular LDPC codes for joint source-channel decoding," IEEE Commun. Lett., vol. 9, no. 12, pp. 1064-1066, Dec. 2005.

[2] G.-C. Zhu and F. Alajaji, "Joint source-channel turbo coding for binary Markov sources," IEEE Trans. Wireless Commun., vol. 5, no. 5, pp. 1065-1075, May 2006.

[3] M. Fresia, F. Perez-Cruz, H. V. Poor, and S. Verdu, "Joint source and channel coding," IEEE Signal Process. Mag., vol. 27, no. 6, pp. 104113, Nov. 2010

[4] J. He, L. Wang, and P. Chen, "A joint source and channel coding scheme base on simple protograph structured codes," in proc. of ISCIT, Oct. 2012, pp. 65-69.

[5] H. Wu, L. Wang, S. Hong, and J. He, "Performance of joint sourcechannel coding based on protograph LDPC codes over Rayleigh fading channels," IEEE Commun. Lett., vol. 18, no. 4, pp. 652-655, Apr. 2014.

[6] T. Bruggen and P. Vary, "Unequal error protection by modulation with unequal power allocation," IEEE Commun. Lett., vol. 9, no. 6, pp. 484 486, Jun. 2005.

[7] F. Cabarcas, R. D. Souza, and J. Garcia-Frias, "Turbo coding of strongly nonuniform memoryless sources with unequal energy allocation and PAM signaling," IEEE Trans. Signal Process., vol. 54, no. 5, pp. 19421946, May 2006.

[8] I. Ochoa, P. M. Crespo, and M. Hernaez, "LDPC codes for non-uniform memoryless sources and unequal energy allocation," IEEE Commun. Lett., vol. 14, no. 9, pp. 794-796, Sep. 2010.

[9] D. Divsalar, S. Dolinar, C. R. Jones, and K. Andrews, "Capacityapproaching protograph codes," IEEE J. Sel. Areas Commun., vol. 27, no. 6, pp. 876-888, Aug. 2009.

[10] X.-Y. Hu, E. Eleftheriou, and D. M. Arnold, "Regular and irregular progressive edge-growth tanner graphs," IEEE Trans. Inf. Theory, vol. 51, no. 1, pp. 386-398, Jan. 2005.

[11] S. ten Brink, G. Kramer, and A. Ashikhmin, "Design of low-density parity-check codes for modulation and detection," IEEE Trans. Commun., vol. 52, no. 4, pp. 670-678, Apr. 2004. 